\title{
A bioelectrical impedance phase angle measuring system for assessment of nutritional status
}

\author{
Guanghao Zhang ${ }^{\mathrm{a}}$, Xiaolin Huo ${ }^{\mathrm{a}, *}$, Changzhe $\mathrm{Wu}^{\mathrm{a}}$, Cheng Zhang ${ }^{\mathrm{a}}$ and Zhongping Duan ${ }^{\mathrm{b}}$ \\ ${ }^{a}$ Beijing Key Laboratory of Bioelectromagnetism, Institute of Electrical Engineering, Chinese \\ Academy of Sciences, No. 6 Beiertiao, Zhongguancun, Beijing, China \\ ${ }^{b}$ Artificial Liver Center, Beijing You'an Hospital, Capital University of Medical Sceiences, No. 8, Xi \\ Tou Tiao, Youanmen wai, Fengtai District, Beijing, China
}

\begin{abstract}
Bioelectrical impedance phase angle has been recommended as a tool to assess nutrition state, but there are no measuring devices have been specially designed for hospital residents. In this study, a system was established for the measurement of bioelectrical impedance phase angle. The electrical composition, calculation method and measuring method of this system are presented in this paper. Experiments showed excellent performance of this system in measuring impedance made of resistors and capacitors. The designed system was also used to measure the bioelectrical impedance phase angle of both healthy subjects and patients with malnutrition, and the results demonstrated that the phase angle of patients with malnutrition is lower than that of healthy subjects $(P<0.01$ for male and $P<0.05$ for female). These results suggest that phase angle has the potential to be a useful tool for the quantitative assessment of nutritional status.
\end{abstract}

Keywords: Phase angle, bioelectrical impedance, nutritional status

\section{Introduction}

Bioelectrical impedance analysis has been widely used in hospital and research area because it is a safe, non-invasive, convenient and economic technology [1]. Biological tissue can be described as a three-component model, which contains extracellular resistance, $R_{\mathrm{o}}$, intracellular resistance, $R_{\mathrm{i}}$, and membrane capacitance, $C_{\mathrm{m}}$ [2]. $R_{\mathrm{o}}$ is parallelly connected with the series connection of $R_{\mathrm{i}}$ and $C_{\mathrm{m}}$. When an alternate current, $I$, flows in biological tissue, a potential difference, $V$, and a phase shift, $\theta$, occur. The impedance, $Z$, and its phase angle (PhA) can be written as $V / I$ and $\theta$, respectively. These two parameters and frequency of current have been used to predict nutritional risks in hospital residents, in addition to the well-known body component analysis (BCA). However, except impedance and $\mathrm{PhA}$, body component data are acquired through regression equations, which are based on age, gender, height and weight of healthy subjects and are not suitable for patients. Due to the lack of bioe-

\footnotetext{
${ }^{*}$ Corresponding author: Xiaolin Huo, Beijing Key Laboratory of Bioelectromagnetism, Institute of Electrical Engineering, Chinese Academy of Sciences, No. 6 Beiertiao, Zhongguancun, Beijing, China. Tel.: 86-10-82547242; Fax: 86-10-82547242; E-mail: huoxl@mail.iee.ac.cn.
} 
lectrical impedance data of hospital residents, rough data, which are not based on regression equations, should be used in nutrition assessment to reduce prediction error.

Previous researches have proved that the impedance and $\mathrm{PhA}$ of $50 \mathrm{kHz}$ carry both intra- and extracellular information. Moreover, $\mathrm{PhA}$ is more appropriate than impedance for the assessment of nutritional status, because $\mathrm{PhA}$ presents the degree of cellular health [3,4]. In healthy persons, PhA ranges from $5^{\circ}$ to $7^{\circ}[5]$, and in athletes it may reach $9.5^{\circ}$ [6]. Low PhA indicates pathological membrane state and function.

In patients suffering from malnutrition, $\mathrm{PhA}$ can reflect the early water shift from intracellular to extracellular space [7-9]. Thus, disease related malnutrition is associated with electrical changes, which can be measured through bioelectrical impedance analysis [10]. Several researches have proved the close relation between $\mathrm{PhA}$ and nutrition state. In hemodialysis patients and patients with renal or gastrointestinal disease, PhA exhibits a negative correlation with the Subjective Global Assessment (SGA) score $[10,11]$. PhA indeed correlates with various indices of functional (e.g., $r=0.53$ with grip strength in liver cirrhosis, $r=0.4$ with knee extension strength and $r=0.35$ with Barthel Index in elderly institutionalized subjects) and nutritional (e.g., $r=0.6$ with albumin, $r=0.53$ with SGA) status $[10,12]$. The improvement of nutritional status is also accompanied by a rise in $\mathrm{PhA}$. Studies show that in patients with Anorexia Nervosa, PhA increased by $0.6^{\circ}$ after 15 weeks of successful nutritional therapy, and $1.1^{\circ}$ in the stable refed state $[13,14]$. In malnourished patients with benign gastrointestinal disease, $\mathrm{PhA}$ increased by $0.34^{\circ} \pm 0.91^{\circ}$ after three months of nutritional intervention [15].

Although PhA has a considerable potential in nutritional status evaluation, currently available instruments used for PhA measurement are only impedance analyzer and body component measuring apparatus. Impedance analyzer is not specifically designed for the measurement of bioelectrical impedance, while body component measuring apparatus lacks PhA measuring accuracy because it targets mainly body components of normal people. In order to design a bioelectrical impedance measuring system with a particular focus on $\mathrm{PhA}$, and to verify its function in hospital residents, the present study was conducted. PhA and bioelectrical impedance of patients with malnutrition and healthy subjects were measured, using the designed system. The measured $\mathrm{PhA}$ of patients were compared with those of healthy subjects as well as previously published data. Possible future work is also discussed in this paper.

\section{Materials and methods}

\subsection{Electrical schematic of the system}

The bioelectrical impedance measuring system designed in this study is based on AD5933, a highprecision impedance converter system solution that combines an on-board frequency generator with a 12-bit, 1 MSPS, analog-to-digital converter (ADC). The electrical schematic of the system is shown in Figure 1.

AD5933 and MAX4684, an analog switch, are controlled by PIC16F873A, a microcontroller provided by Microchip Technology Inc. AD5933 outputs a constant voltage, $V_{\mathrm{o}}$, between the pins of VIN and VOUT. First, VIN and VOUT were set to connect with NO1 and NO2, respectively. The calibration resistance, $R_{0}$, is connected to the RFB pin of AD5933. Therefore, the input signal to inner ADC of AD5933 is $V_{\mathrm{s} 1}=-V_{\mathrm{0}} R_{\mathrm{FB}} / R_{0}$, according to the datasheet of AD5933. Second, VIN and VOUT were set to connect with NC2 and NO1, respectively. The constant voltage output at VOUT is converted to a constant current by the voltage to current transform circuit, which consists of an operational amplifier, 


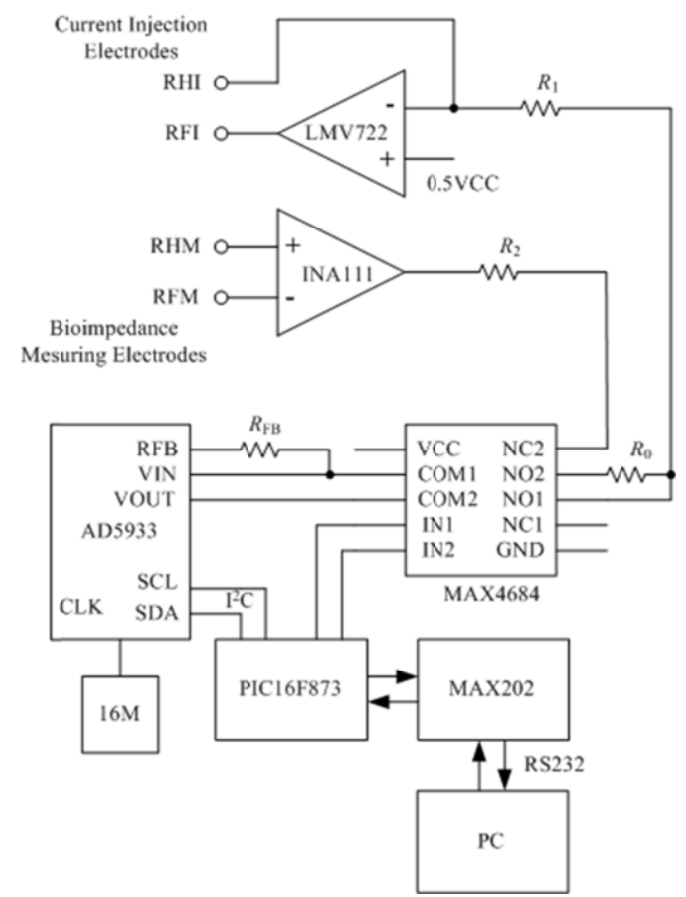

Fig. 1. Electrical schematic of the designed bioelectrical impedance measuring system.

LMV722, a current-limiting resistance, $R_{1}$, and the bioelectrical impedance, $Z$, between two current injecting electrodes, RHI and RFI. Therefore, the constant current flow in $Z$ is $I=V_{\mathrm{o}} / R_{1}$. A different amplifier, INA111, with a gain of $G$, measures the voltage between the two measuring electrodes, RHM and RFM. Therefore, the output of INA111 is $G Z V_{0} / R_{1}$. The input signal to inner ADC of AD5933 is $V_{\mathrm{s} 2}=-G Z R_{\mathrm{FB}} V_{0} /\left(R_{1} R_{2}\right)$.

Then, the information containing bioelectrical impedance and $\mathrm{PhA}$ is sent to microcontroller through $\mathrm{I}^{2} \mathrm{C}$ interface. The computer reads the data in microcontroller through $\mathrm{RS} 232$ interface and calculates the impedance and PhA of the bioelectrical impedance. The frequency of $V_{\mathrm{o}}$ ranges from 1 $\mathrm{kHz}$ to $100 \mathrm{kHz}$ by the method of frequency sweep with a step of $1 \mathrm{kHz}$.

It is important to make sure that the current injected to the human body is in the safe range. In this system, the output peak to peak value of $V_{\mathrm{o}}$ was set to $3 \mathrm{~V}$. A $1.5 \mathrm{k} \Omega$ resistor was chosen as the current limiting resistor. Thus, the effective current injected into the human body is about $0.71 \mathrm{~mA}$.

\subsection{Calculation of impedance and phase angle}

The response signal from the bioelectrical impedance is sampled by the on-board ADC and DFTprocessed by an on-board DSP engine in AD5933. The discrete Fourier transformation (DFT) algorithm returns both a real $\left(R_{\mathrm{z}}\right)$ and an imaginary $\left(I_{\mathrm{z}}\right)$ data-word at a given frequency. So the twice output data of AD5933 are

$$
R_{z 1}+j I_{z 1}=\operatorname{DFT}\left(V_{s 1}\right)=\operatorname{DFT}\left[-V_{o} R_{F B} / R_{0}\right]
$$




$$
R_{z 2}+j I_{z 2}=\operatorname{DFT}\left(V_{s 2}\right)=\operatorname{DFT}\left[-G Z R_{F B} V_{0} /\left(R_{1} R_{2}\right)\right] .
$$

Since the right side of Eq. (1) has no imagery part, it can be rewritten as

$$
R_{z 1}=\operatorname{DFT}\left(V_{s 1}\right)=R_{F B} / R_{0} \cdot \operatorname{DFT}\left[-V_{o}\right],
$$

while Eq. (2) can be expressed as

$$
K e^{j \alpha}=G Z R_{F B} /\left(R_{1} R_{2}\right) D F T\left[-V_{0}\right]
$$

where

$$
\begin{aligned}
& K^{2}=R_{z 2}^{2}+I_{z 2}^{2}, \\
& \alpha=\operatorname{atan}\left(I_{z 2} / R_{z 2}\right) .
\end{aligned}
$$

In order to simplify the calculation, the values of $R_{0}, R_{1}, R_{2}$ and $G$ need to be carefully chosen to make the value of $R_{1} R_{2} /\left(G R_{0}\right)$ an integral. Finally, the values of $R_{0}, R_{1}, R_{2}$ and $G$ were chosen as 1.5 $\mathrm{k} \Omega, 1.5 \mathrm{k} \Omega, 1 \mathrm{k} \Omega$ and 1 . Hence, the bioelectrical impedance, $Z$, and its $\mathrm{PhA}, \theta$, can be calculated with Eqs. (3)-(6) as

$$
\begin{aligned}
& |Z|=1000 K / R_{z 1}, \\
& \theta=\alpha .
\end{aligned}
$$

All these calculation were done by a computer program written in $\mathrm{C}++$ programming language. Only the impedance and $\mathrm{PhA}$ of $50 \mathrm{kHz}$ were used and other data were saved for future use. When the measurement was carried out, $R_{\mathrm{z} 1}$ was firstly obtained from the known resistance $R_{0}$. Then, $I_{\mathrm{z} 2}$ and $R_{\mathrm{z} 2}$ were acquired from the bioelectrical impedance to be measured. Finally, $Z$ and $\theta$ were calculated with the computer program.

\subsection{Subjects and measuring method}

The study included fifteen healthy adult subjects, 9 male and 6 female, recruited from our lab, and they were divided into two groups by gender. Patients with malnutrition recruited from hospital residents, including 9 male and 6 female, were also divided into two groups by gender. Informed consents were obtained from all volunteers prior to the measurement.

During the measurement, each subject laid flat on their back on a bed. Adhesive gel electrodes (3M RedDot) were placed at defined anatomical sites on the dorsal surfaces of the subject's hand, wrist, 
ankle, and foot according to previous studies as follows: the proximal edge of the first measuring electrode was attached from an imaginary line at styloid process of the ulna and the distal edge of the finger current injecting electrode on an imaginary line from the middle of the metacarpophalangeal joints of the index and middle fingers. The distal edge of the toe current injecting electrode was placed from an imaginary line through the middle of the metatarsophalangeal joints of the second and third toes. The proximal edge of the ankle joint measuring electrode was attached along a line through the highest points of the outer and inner ankle bones. Detailed description of the measurement procedure has been given by Kushner [16].

For each subject, the bioelectrical impedance and $\mathrm{PhA}$ were measured three times, and the mean value was adopted for analysis. All data are presented in the form of mean \pm SD in this paper. The statistical analysis was performed by using SPSS. The PhA and impedance of different groups were compared through analysis of variance (ANOVA). A $P<0.05$ was considered indicating a significant difference.

\section{Results}

In order to verify the accuracy of the system, resistors and capacitors with known values were used for experiments. The values of these components were selected to mimic the $\mathrm{PhA}$ of people. Table 1 shows the values of resistors and capacitors measured by digital multimeter, as well as the impedance and $\mathrm{PhA}$ obtained by the designed system. Obviously, the system has a good performance when measuring known impedance.

Table 2 and Figure 2 show the values of impedance and PhA of healthy subjects and patients with malnutrition. In healthy subjects, the mean PhA of male is higher than that of female $(P<0.05)$. This is consistent with the findings of previous studies $[5,17,18]$. However, there is no significant difference between the PhA of male and female patients with malnutrition $(P>0.05)$. The mean PhA of both male and female patients with malnutrition are lower than those of healthy subjects $(P<0.01$ for male, $P<0.05$ for female). This is also consistent with the findings of previous studies [17-19]. There is no significant difference between the impedance of any two groups $(P>0.05)$. Considering the small sample size, only gender difference was considered.

Table 1

Impedance consists of resistors and capacitors

\begin{tabular}{llll}
\hline & Group 1 & Group 2 & Group 3 \\
\hline $\operatorname{Ri}(\Omega)$ & 1000 & 1000 & 750 \\
\hline $\operatorname{Ro}(\Omega)$ & 750 & 500 & 500 \\
\hline $\mathrm{Cm}(\mu \mathrm{F})$ & 1 & 1 & 2 \\
\hline $\operatorname{Real~Z}(\Omega)$ & 429 & 333 & 300 \\
\hline Real $\operatorname{PhA}\left({ }^{\circ}\right)$ & 7.8 & 6.1 & 4.9 \\
\hline Measured $\mathrm{Z}(\Omega)$ & 435 & 338 & 302 \\
\hline Measured $\mathrm{PhA}\left({ }^{\circ}\right)$ & 7.8 & 6.2 & 4.9 \\
\hline
\end{tabular}

Note: Real $\mathrm{Z}$ and Real PhA denote the values calculated from the values measured by digital multimeter. Measured $\mathrm{Z}$ and Measured $\mathrm{PhA}$ denote the values obtained from the system presented in this paper. 
Table 2

Impedance and $\mathrm{PhA}$ of healthy subjects and patients with malnutrition

\begin{tabular}{llll}
\hline & Healthy Subjects & Patients & $P$ \\
\hline Impedance $(\Omega)$, Male & $353 \pm 19.6$ & $362 \pm 43.1$ & $>0.05$ \\
\hline $\operatorname{PhA}\left({ }^{\circ}\right)$, Male & $6.84 \pm 1.05$ & $3.89 \pm 1.34$ & $<0.01$ \\
\hline Impedance $(\Omega)$, Female & $413 \pm 27.6$ & $334 \pm 75.5$ & $>0.05$ \\
\hline $\operatorname{PhA}\left({ }^{\circ}\right)$, Female & $4.95 \pm 1.08$ & $2.20 \pm 2.14$ & $<0.05$ \\
\hline
\end{tabular}

Note: The difference between populations is considered significant when $P$ is below the level of 0.05 .

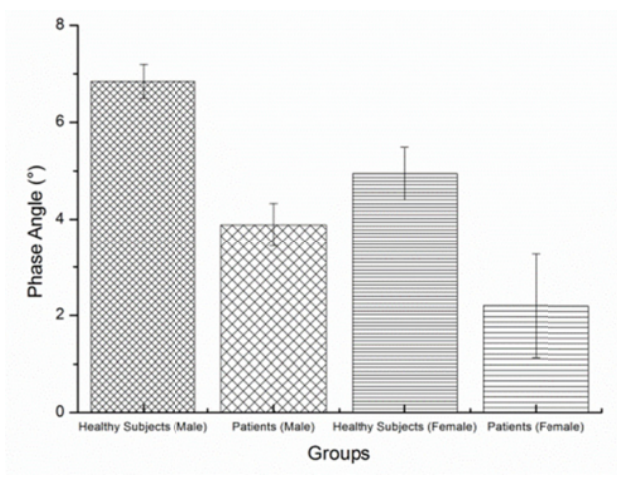

A Phase angle

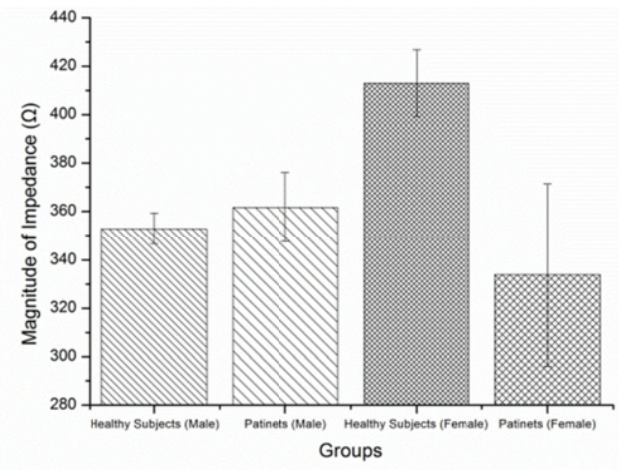

B Magnitude of impedance

Fig. 2. Impedance and $\mathrm{PhA}$ of Healthy subjects and Patients with malnutrition.

\section{Conclusion and discussion}

This study was conducted to design a bioelectrical impedance measuring system that measures the impedance and $\mathrm{PhA}$ of patients of malnutrition. The reliability of the designed system was proved by its excellent performance when it was used to measure impedance that was made of resistor and capacitor.

The measurement of bioelectrical impedance with the system is different from the measurement of $R C$ components in several ways. First, the interface between electrodes and human skin is an important factor because the contact resistance may affect the measuring results seriously, especially the $\mathrm{PhA}$. In this paper, we used adhesive gel electrodes provided by $3 \mathrm{M}$, for both current injecting electrodes and measuring electrodes. Changes of current injecting electrodes manufacturer would lead to great changes of the measured $\mathrm{PhA}$ while changes of measuring electrodes cause only minor changes of PhA. Meanwhile, the type of the electrodes has no influence on the impedance. These phenomena demonstrate that the importance of electrode-skin interface in measuring $\mathrm{PhA}$. To select appropriate gel type, contact area and connection method, a model of this interface should be built and studied. Although the 3M RedDot electrodes showed good performance in this study, they are initially designed for measurement of electrocardiogram instead of bioelectrical impedance and $\mathrm{PhA}$. Therefore, electrodes specially designed for the measurement of PhA are needed. Moreover, the positioning of 
electrodes is another important factor influencing the measured PhA because slight movement of any one of the electrodes would lead to changes of $\mathrm{PhA}$, as well as impedance. Hence, during $\mathrm{PhA}$ measurement, it is also important to follow the already established process for determining electrodes positions to improve comparability between various kinds of studies.

In this study, only the impedance and $\mathrm{PhA}$ of $50 \mathrm{kHz}$ were used. Although it has been widely accepted that $50 \mathrm{kHz}$ is a proper frequency in nutrition assessment, more rough data of other frequencies may increase the accuracy of diagnosis. It is easy to obtain the extracellular resistance, $R_{0}$, by using low frequency injecting current. When $R_{\mathrm{o}}$ is known, the intra- cellular resistance, $R_{\mathrm{i}}$, could be obtained by injecting high frequency current. Together with the impedance of $50 \mathrm{kHz}$, membrane capacitance, $C_{\mathrm{m}}$, could also be calculated. Assuming that the three components do not change with frequency, impedance and $\mathrm{PhA}$ of the whole frequency band can be calculated and described in a diagram. Such data and diagrams will greatly help in assessing nutrition state.

Although the numbers of healthy subjects and patients in this study were small, a certain relationship between PhA and nutrition state was revealed. Patients with a better nutrition state showed relatively higher $\mathrm{PhA}$ than the patients with severe malnutrition, such as patients with liver cancer or hepatic failure. This phenomenon suggests that $\mathrm{PhA}$ has the potential to be a quantitative index in assessing nutrition state while current assessing methods such as SGA contain many subjective factors. Therefore, a large number of subjects with malnutrition in hospital residents will be recruited in our future study. Meanwhile, the control group will include healthy subjects of more diverse gender, age, height and weight. However, phase angle can be influenced by many factors, and the limitation of this measuring system and factors may influence the measurement. For example, skin moisture, charges produced by friction of clothes and slight movement of subjects all affect the measuring results. Moreover, phase angle is closely associated with Body Mass Index (BMI). In other words, patients with higher BMI might have the same phase angle with healthy persons with lower BMI. In order to overcome these limitations in PhA measurement and data analysis, the circuit and electrodes system should be improved and standard measuring process and appropriate data processing methods should also be established. Finally, a nutrition state assessing method based on PhA is expected to be established and implemented in clinical trials.

\section{Acknowledgement}

This research was supported by the National Natural Science Foundation of China, No. 51307166. The study was partly conducted at the Beijing You'an Hospital with the help of Doctor Ming Kong and Doctor Li Zhou.

\section{References}

[1] A.C. Buchholz, C.F. Mcgillivray and P.B. Pencharz, The use of bioelectric impedance analysis to measure fluid compartments in subjects with chronic paraplegia, Archives of Physical Medicine and Rehabilitation 84 (2003), 854-861.

[2] L. Hui and L.W. Ding, Low-power and portable design of bioelectrical impedance measurement system, 2010 WASE International Conference on Information Engineering (ICIE) 3 (2010), 38-41.

[3] J. Mattar, Application of total body bioimpedance to the critically ill patient, New Horizons (Baltimore, Md) 4 (1996), 493-503.

[4] H.J. Zdolsek, O.A. Lindahl and S.J. Berg, Non-invasive assessment of fluid volume status in the interstitium after haemodialysis, Physiological Measurement 21 (2000), 211-220. 
[5] A. Bosy-Westphal, S. Danielzik, H.P. Dörhöfer et al., Phase angle from bioelectrical impedance analysis: Population reference values by age, sex, and body mass index, Journal of Parenteral and Enteral Nutrition 30 (2006), 309-316.

[6] A.G. Torres, K.J. Oliveira, A.V. Oliveira-Junior et al., Biological determinants of phase angle among Brazilian elite athletes, Proceedings of the Nutrition Society 67 (2008), E332.

[7] H. Shizgal, The effect of malnutrition on body composition, Surgery, Gynecology \& Obstetrics 152 (1981), $22-26$.

[8] O. Selberg and D. Selberg, Norms and correlates of bioimpedance phase angle in healthy human subjects, hospitalized patients, and patients with liver cirrhosis, European Journal of Applied Physiology 86 (2002), 509-516.

[9] F.W. Guglielmti, T. Mastronuzzi, L. Pietrini et al., The RXc graph in evaluating and monitoring fluid balance in patients with liver cirrhosis, Annals of the New York Academy of Sciences 873 (1999), 105-111.

[10] K. Noman, C. Smoliner, A. Kilber et al., Disease-related malnutrition but not underweight by BMI is reflected by disturbed electric tissue properties in the bioelectrical impedance vector analysis, British Journal of Nutrition 100 (2008), $590-595$

[11] C. Oliveira, M. Kubrusly, R.S. Mota et al., The phase angle and mass body cell as markers of nutritional status in hemodialysis patients, Journal of Renal Nutrition 20 (2010), 314-320.

[12] S.M. Gunn, J.A. Halbert, L.C. Giles et al., Bioelectrical phase angle values in a clinical sample of ambulatory rehabilitation patients, Dynamic Medicine 7 (2008), 14.

[13] C. Mika, B. Herpertz-Dahlmann, M. Heer et al., Improvement of nutritional status as assessed by multifrequency BIA during 15 weeks of refeeding in adolescent girls with anorexia nervosa, The Journal of Nutrition 134 (2004), 30263030 .

[14] L. Scalfi, M. Marra, A. Caldara et al., Changes in bioimpedance analysis after stable refeeding of undernourished anorexic patients, International Journal of Obesity 23 (1999), 133-137.

[15] K. Norman, H. Kirchner, M. Freudenreich et al., Three month intervention with protein and energy rich supplements improve muscle function and quality of life in malnourished patients with non-neoplastic gastrointestinal disease: A randomized controlled trial, Clinical Nutrition 27 (2008), 48-56.

[16] R.F. Kushner, Bioelectrical impedance analysis: A review of principals and applications, Am. Coll. Nutr. 11 (1992), 199-209.

[17] U.G. Kyle, E.P. Soundar, L. Genton and C. Pichard, Can phase angle determined by bioelectrical impedance analysis assess nutritional risk? A comparison between healthy and hospitalized subjects, Clinical Nutrition 31 (2012), 875-881.

[18] N. Stobaus, M. Pirlich and L. Valentini, Determinants of bioelectrical phase angle in disease, British Journal of Nutrition 107 (2012), 1217-1220.

[19] U.G. Kyle, L. Genton and C. Pichard, Low phase angle determined by bioelectrical impedance analysis is associated with malnutrition and nutritional risk at hospital admission, Clinical Nutrition 32 (2013), 294-299. 